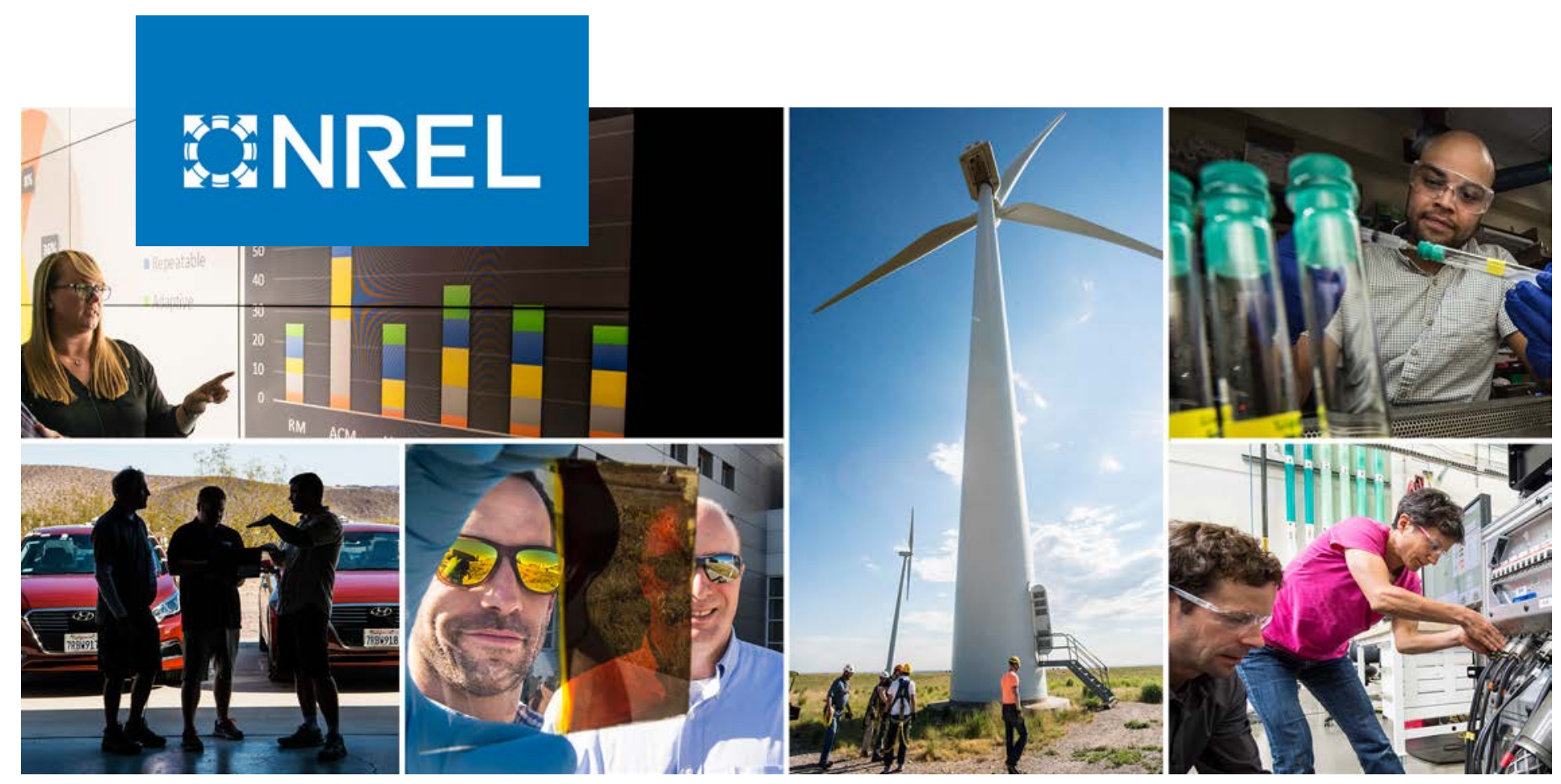

\title{
Dispatchable Virtual Oscillator Control for Decentralized Inverter-Dominated Power Systems: Analysis and Experiments
}

\section{Preprint}

Gab-Su Seo, ${ }^{1}$ Marcello Colombino, ${ }^{1}$ Irina Subotic, ${ }^{2}$ Brian Johnson, ${ }^{3}$ Dominic Gross, ${ }^{2}$ and Florian Dorfler ${ }^{2}$

${ }^{1}$ National Renewable Energy Laboratory

${ }^{2}$ ETH Zurich

${ }^{3}$ University of Washington

Presented at the 2019 IEEE Applied Power Electronics Conferences (IEEE PELS)

Anaheim, California

March 17-21, 2019

NREL is a national laboratory of the U.S. Department of Energy Office of Energy Efficiency \& Renewable Energy

Operated by the Alliance for Sustainable Energy, LLC

This report is available at no cost from the National Renewable Energy Laboratory (NREL) at www.nrel.gov/publications.
Conference Paper

NREL/CP-5D00-72774

September 2019 


\title{
GNREL
}

\section{Dispatchable Virtual Oscillator Control for Decentralized Inverter-Dominated Power Systems: Analysis and Experiments}

\section{Preprint}

\author{
Gab-Su Seo, ${ }^{1}$ Marcello Colombino, ${ }^{1}$ Irina Subotic, ${ }^{2}$ \\ Brian Johnson, ${ }^{3}$ Dominic Gross, ${ }^{2}$ and Florian Dorfler ${ }^{2}$ \\ ${ }^{1}$ National Renewable Energy Laboratory \\ ${ }^{2}$ ETH Zurich \\ ${ }^{3}$ University of Washington
}

\section{Suggested Citation}

Seo, Gab-Su, Marcello Colombino, Irina Subotic, Brian Johnson, Dominic Gross, and Florian Dorfler. 2019. Dispatchable Virtual Oscillator Control for Decentralized Inverter-Dominated Power Systems: Analysis and Experiments: Preprint. Golden, CO: National Renewable Energy Laboratory. NREL/CP-5D00-72274. https://www.nrel.gov/docs/fy19osti/72774.pdf.

(C) 2019 IEEE. Personal use of this material is permitted. Permission from IEEE must be obtained for all other uses, in any current or future media, including reprinting/republishing this material for advertising or promotional purposes, creating new collective works, for resale or redistribution to servers or lists, or reuse of any copyrighted component of this work in other works.

NREL is a national laboratory of the U.S. Department of Energy Office of Energy Efficiency \& Renewable Energy Operated by the Alliance for Sustainable Energy, LLC

This report is available at no cost from the National Renewable Energy Laboratory (NREL) at www.nrel.gov/publications.

Contract No. DE-AC36-08GO28308
Conference Paper

NREL/CP-5D00-72774

September 2019

National Renewable Energy Laboratory 15013 Denver West Parkway Golden, CO 80401

303-275-3000 • www.nrel.gov 


\section{NOTICE}

This work was authored in part by the National Renewable Energy Laboratory, operated by Alliance for Sustainable Energy, LLC, for the U.S. Department of Energy (DOE) under Contract No. DE-AC36-08GO28308. Funding provided by U.S. Department of Energy Office of Energy Efficiency and Renewable Energy Solar Energy Technologies Office. The views expressed herein do not necessarily represent the views of the DOE or the U.S. Government. The U.S. Government retains and the publisher, by accepting the article for publication, acknowledges that the U.S. Government retains a nonexclusive, paid-up, irrevocable, worldwide license to publish or reproduce the published form of this work, or allow others to do so, for U.S. Government purposes.

This report is available at no cost from the National Renewable Energy Laboratory (NREL) at www.nrel.gov/publications.

U.S. Department of Energy (DOE) reports produced after 1991 and a growing number of pre-1991 documents are available free via www.OSTI.gov.

Cover Photos by Dennis Schroeder: (clockwise, left to right) NREL 51934, NREL 45897, NREL 42160, NREL 45891, NREL 48097, NREL 46526.

NREL prints on paper that contains recycled content. 


\title{
Dispatchable Virtual Oscillator Control for Decentralized Inverter-dominated Power Systems: Analysis and Experiments
}

\author{
Gab-Su Seo*, Marcello Colombino*, Irina Subotic ${ }^{\dagger}$, Brian Johnson ${ }^{\ddagger}$, Dominic Groß ${ }^{\dagger}$, and Florian Dörfler ${ }^{\dagger}$ \\ * Power Systems Engineering Center, National Renewable Energy Laboratory, Golden, CO 80401, USA \\ Email: Gabsu.Seo@nrel.gov, Marcello.Colombino@nrel.gov \\ ${ }^{\dagger}$ Automatic Control Laboratory, ETH Zurich, Zurich 8092, Switzerland \\ Email: subotici@ethz.ch, grodo@ethz.ch,dorfler@ethz.ch \\ $\ddagger$ Department of Electrical and Computer Engineering, University of Washington, Seattle, WA 98195, USA \\ Email: brianbj@uw.edu
}

\begin{abstract}
This paper presents an analysis and experimental validation of dispatchable virtual oscillator control (dVOC) for inverter-dominated power systems. dVOC is a promising decentralized control strategy that requires only local measurements to induce grid-forming behavior with programmable droop characteristics. It is dispatchable-i.e., the inverters can vary their power generation via user-defined power set-points and guarantees strong stability. To verify its feasibility, a testbed comprising multiple dVOC-programmed inverters with transmission line impedances is designed. With an embedded synchronization strategy, the dVOC inverters are capable of dynamic synchronization, black start operation, and transient grid voltage regulation with dynamic load sharing, and real-time-programmable droop characteristics for backward compatibility. All these features are experimentally verified.
\end{abstract}

Index Terms-Microgrid, droop control, nonlinear control, synchronization, decentralized control, grid-forming control, voltage source inverters

\section{INTRODUCTION}

The electric power grid is undergoing exceptional changes with increasing penetration of inverter-based renewable generation [1], [2]. As of now, stability and system-wide synchronization of the grid is achieved with traditional synchronous generators and their controls. Conventionally, power inverters are controlled in a "grid following" fashion. This means that they are programmed to estimate the (already stable) grid frequency and regulate their injected current to track pre-determined power set-points. In a purely inverter-based grid, without synchronous generators, these control strategies are no longer suitable as they cannot maintain stability and

This work was authored in part by the National Renewable Energy Laboratory, operated by Alliance for Sustainable Energy, LLC, for the U.S Department of Energy (DOE) under Contract No. DE-AC36-08GO28308. Funding was provided in part by the DOE Office of Energy Efficiency and Renewable Energy Solar Energy Technologies Office grant number DEEE0000-1583. This project has received funding in part from the European Union's Horizon 2020 research and innovation program under grant agreement $\mathrm{No}^{\circ}$ 691800. This paper reflects only the authors' views. The views expressed in the article do not necessarily represent the views of the DOE, the U.S Government, or the European Commission. The European Commission is not responsible for any use that may be made of the information it contains. synchronization. To obviate this problem and envision a stable inverter-based grid, grid-forming control methods have been proposed [3]-[5]. A grid-forming inverter is not limited to power tracking but acts as a controlled voltage source that can change its power output (thanks to storage or curtailment), and is controlled to contribute to the stability of the grid. It is highly desirable that inverter control strategies are decentralized, i.e., they rely on local measurements only [6] as this allows for plug-and play capabilities. We envision that, with the advent of renewable generation and grid-forming control, portions of the grid can be islanded if needed without loosing synchronization and load sharing. This improves modularity and thus resiliance of the grid to natural disasters or cyberphysical attacks.

Most of the common approaches of grid-forming control focus on droop control [7]-[9]. Its simple implementation and backward compatibility [10], make droop control a desirable solution accepted by utilities and practitioners. However, the associated phasor models are well-defined only near the synchronous steady-state [11]. Other popular approaches are based on mimicking the physical characteristics and controls of synchronous machines [12]-[14]. While strategies based on machine-emulation and droop are compatible with legacy power systems, they use an energy conversion interface (an inverter) with fast actuation but almost no inherent energy storage to mimic another interface (a generator) with slow actuation but significant energy storage (in the form of rotational inertia). It is unclear if this is a viable option, especially taking into account the limited energy storage and tight over-current constraints of power inverters [15].

Recently proposed solutions based on virtual oscillator control (VOC) feature enhanced dynamic performance and maintain an embedded droop control law [16] close to steadystate. This implies a superior voltage regulation performance with respect to standard droop control, while keeping load sharing capabilities [11], [17]. In addition to these benefits, ease of synchronization allows VOC to be a promising candidate for microgrids; however, it is unclear how to dispatch 


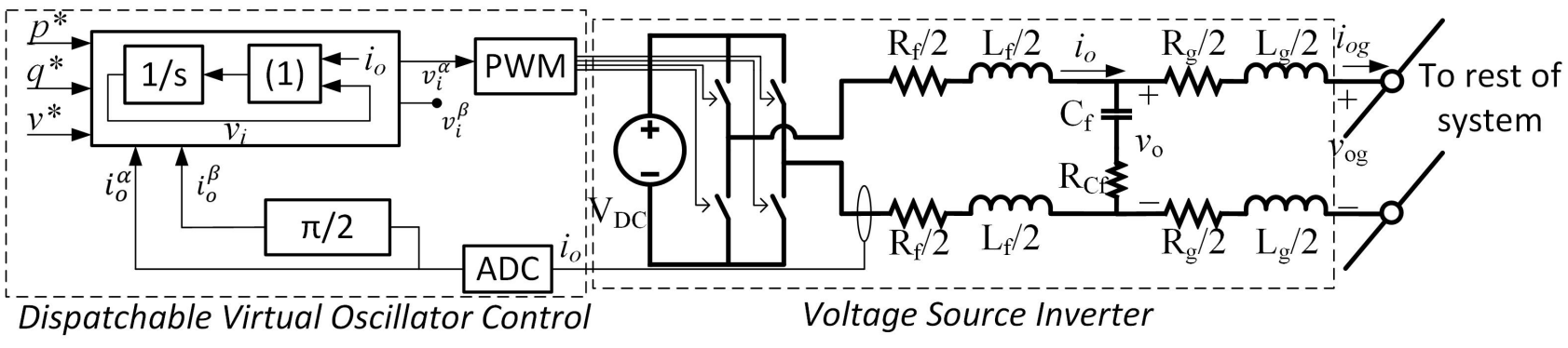

Fig. 1. Schematic of dVOC inverter for decentralized inverter-dominant grid

VOC inverters-i.e., reconfigure the inverters' power injections as they have no programmable power set-points. This paper investigates a recently proposed dispatchable virtual oscillator control (dVOC) with the following desirable features:

i) dVOC is dispatchable, i.e. it allows for the user to specify power set-points for each inverter.

ii) Given no set-points, dVOC subsumes VOC control and therefore it inherits all its favorable dynamical properties.

iii) Under the assumption that the set-points are consistent with the $\mathrm{AC}$ power flow equations and other technical assumptions, dVOC renders the grid globally asymptotically stable with respect to the desired solution of the AC power-flow [18].

The contribution of this paper is twofold: first, we analyze the inherent droop characteristics that dVOC shows around a synchronous trajectory. This allows practitioners who are accustomed to working with droop controllers to understand the effect of the dVOC parameters on its steady-state behavior. Finally, we present an experimental validation of dVOC's feasibility for an inverter-based microgrid, where we validate its stability, load sharing, and droop properties in a twoinverter system.

The paper is structured as follows. In Section II we introduce the dVOC control; in Section III, we derive the dVOC droop characteristics; in Section IV, we present the experimental setup and preliminary validation results for dVOC, and in Section $\mathrm{V}$ we conclude by summarizing the results and outlining the planned future work.

\section{DVOC FOR INVERTERS}

This section reviews the basics of dVOC. A detailed description of the control strategy can be found in [18], [19].

\section{A. Dispatchable Virtual Oscillator Control}

Dispatchable VOC is a dencentralized "grid forming" control strategy designed to achieve synchronization of an inverter-dominant grid, while maintaining a level of control on the power injections and voltage level of each inverter. When applying dVOC, each inverter monitors its output current $i_{o}$ and, using a PWM strategy, regulates its terminal voltage vector $v_{i}=\left[v_{i}^{\alpha}, v_{i}^{\beta}\right]^{\top}$, (in the $\alpha-\beta$ coordinate frame) to follow the dVOC control law:

$$
\frac{\mathrm{d}}{\mathrm{d} t} v_{i}=\omega_{0} J v_{i}+\eta\left(K_{i} v_{i}-R(\kappa) i_{o, i}+\alpha \phi_{i}\left(v_{i}\right) v_{i}\right),
$$

where $i_{o, i}=\left[i_{o, i}^{\alpha}, i_{o, i}^{\beta}\right]^{\top}$ is the measurement of the inverter current (for single phase signals the $\beta$ component is reconstructed using a Hilbert transform), $\omega_{0}$ is the nominal grid frequency, the matrix

$$
R(\kappa):=\left[\begin{array}{cc}
\cos (\kappa) & -\sin (\kappa) \\
\sin (\kappa) & \cos (\kappa)
\end{array}\right]
$$

is a $2 \mathrm{D}$ rotation matrix, $J:=R(\pi / 2)$,

$$
K_{i}:=\frac{1}{v_{i}^{\star 2}} R(\kappa)\left[\begin{array}{cc}
p_{i}^{\star} & q_{i}^{\star} \\
-q_{i}^{\star} & p_{i}^{\star}
\end{array}\right], \quad \phi_{i}\left(v_{i}\right):=\frac{v_{i}^{\star 2}-\left\|v_{i}\right\|^{2}}{v_{i}^{\star 2}},
$$

the operator $\|\cdot\|$ is the Euclidean norm, the quantities $\eta>$ $0, \alpha>0$ and $0 \leq \kappa \leq \pi$ are the design parameters, and $p_{k}^{\star}, q_{k}^{\star}$, and $v_{k}^{\star}$ are the active power, reactive power, and voltage magnitude set-points, respectively. The parameter $\kappa$ can be used to adjust the controller to adapt to the line parameters, $\kappa=0$ corresponds to resistive lines and $\kappa=\pi / 2$ corresponds to inductive lines. Fig. 1 shows a schematic of an inverter implementing dVOC.

\section{B. Interpretation of the dVOC Controller}

Equation (1) represents the dVOC controller introduced in [18]. In order to give an intuitive interpretation to dVOC, we can write (1) as

$$
\frac{\mathrm{d}}{\mathrm{d} t} v_{i}=\omega_{0} J v_{i}+\eta e_{\theta, i}\left(v_{i}, i_{o, i}\right)+\eta \alpha e_{v, i}\left(v_{i}\right),
$$

where $\omega_{0} J v_{i}$ is the standard equation of a harmonic oscillator in rectangular coordinates, $e_{\theta, i}\left(v_{i}, i_{o, i}\right)=K_{i} v_{i}-R(\kappa) i_{o, i}$ represents a "phase error" term (see [19, Sec. II. D.]), and $e_{v, i}\left(v_{i}\right)=\phi_{i}\left(v_{i}\right) v_{i}$ represents a magnitude error term. Note, that the magnitude error vanishes when $\left\|v_{i}\right\|=v_{i}^{\star}$. Moreover, the "phase error" $e_{\theta, i}\left(v_{i}, i_{o, i}\right)$ vanishes when the voltage levels and power injections of the inverters corresponds to the setpoints. To see this note that

$$
\frac{1}{v_{i}^{\star 2}}\left[\begin{array}{cc}
p_{i}^{\star} & q_{i}^{\star} \\
-q_{i}^{\star} & p_{i}^{\star}
\end{array}\right] v_{i}-i_{0, i}=0
$$

whenever $v_{i}^{\top} i_{o, i}=p_{i}^{\star}$ and $v_{i}^{\top} J i_{o, i}=q_{i}^{\star}$. In [19], [20], it is shown that, if all inverters in the grid implement (1), and all set-points are consistent with the $\mathrm{AC}$ power-flow equations, and further technical assumptions are satisfied, the inverterbased grid is (almost) globally asymptotically stable with 


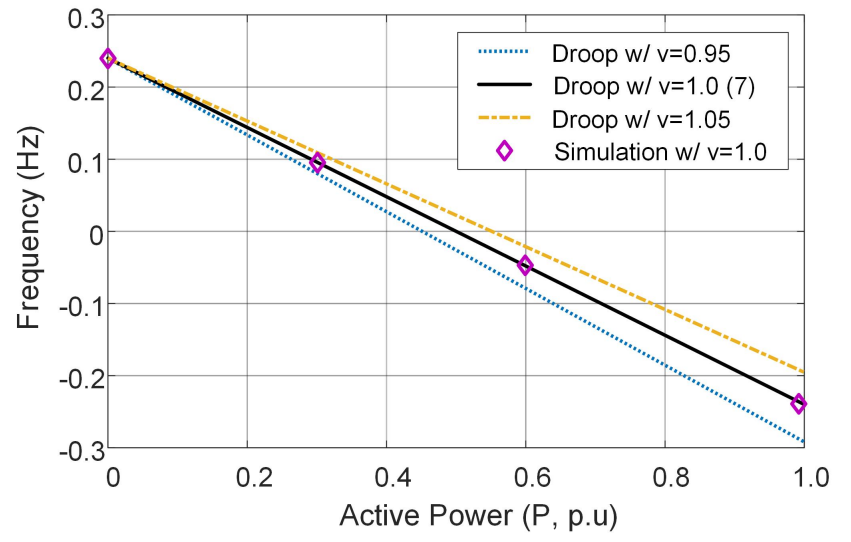

(a)

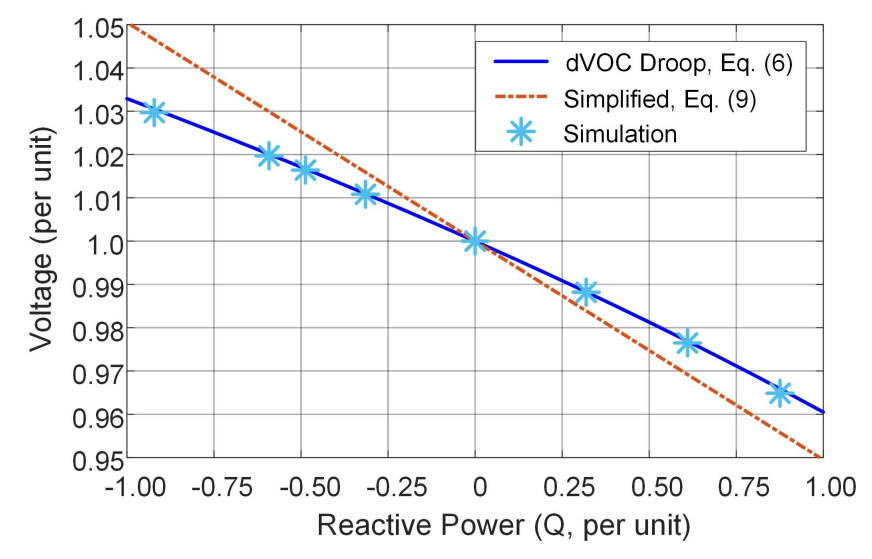

(b)

Fig. 2. Droop characteristics of dVOC: (a) frequency to active power droop, (b) voltage to reactive power droop. We observe that the theoretical droop curves fit very well with high fidelity simulations. Experimental validation of the droop curves is part of our current and future efforts.

respect to the desired power-flows. This means that regardless of the initial conditions, the inverters synchronize and reach the desired set-points. Furthermore, when the set-points are inconsistent with the power-flow equations, dVOC presents droop-like characteristics that enable grid synchronization while also giving trade-offs between power imbalance and reactive power versus frequency and voltage. These droop characteristics will be discussed in depth in the next section.

\section{DRoop Characteristics OF DVOC}

Droop control is the most popular decentralized gridforming control strategy as it is backward compatible with generators and enables power sharing [21]. With conventional droop control, the frequency and magnitude of the voltage of an inverter are determined by active and reactive power mismatches as follows

$$
\begin{gathered}
\frac{\mathrm{d}}{\mathrm{d} t} \theta=\omega_{i}=\omega_{0}+k_{p}\left(p_{i}^{\star}-p_{i}\right), \\
\frac{\mathrm{d}}{\mathrm{d} t}\left\|v_{i}\right\|=-\left\|v_{i}\right\|+v_{i}^{\star}+k_{q}\left(q_{i}^{\star}-q_{i}\right),
\end{gathered}
$$

where $k_{p}$ and $k_{q}$ are droop coefficients. If we consider (1) in polar coordinates [19], we observe that dVOC presents the following nonlinear droop behavior

$$
\begin{aligned}
\frac{\mathrm{d}}{\mathrm{d} t}\left[\begin{array}{c}
\left\|v_{i}\right\| \\
\theta_{i}
\end{array}\right] & =\eta\left[\begin{array}{cc}
\left\|v_{i}\right\| & 0 \\
0 & 1
\end{array}\right] R(\kappa)\left[\begin{array}{c}
\frac{p_{i}^{\star}}{v_{i}^{\star 2}}-\frac{p_{i}}{\left\|v_{i}\right\|^{2}} \\
-\left(\frac{q_{i}^{\star}}{v_{i}^{\star 2}}-\frac{q_{i}}{\left\|v_{i}\right\|^{2}}\right)
\end{array}\right] \\
& +\left[\begin{array}{c}
\frac{\eta \alpha}{v_{i}^{\star 2}}\left(v_{i}^{\star 2}-\left\|v_{i}\right\|^{2}\right)\left\|v_{i}\right\| \\
\omega_{0}
\end{array}\right] .
\end{aligned}
$$

Choosing $\kappa=\pi / 2$ in (5) yields the final dVOC droop ( $V-$ $Q, \omega-P)$

$$
\begin{aligned}
\frac{\mathrm{d}}{\mathrm{d} t} \theta_{i} & =\omega_{0}+\eta\left(\frac{p_{i}^{\star}}{v_{i}^{\star 2}}-\frac{p_{i}}{\left\|v_{i}\right\|^{2}}\right) \\
\frac{\mathrm{d}}{\mathrm{d} t}\left\|v_{i}\right\| & =\eta\left(\frac{q_{i}^{\star}}{v_{i}^{\star 2}}-\frac{q_{i}}{\left\|v_{i}\right\|^{2}}\right)\left\|v_{i}\right\| \\
& +\frac{\eta \alpha}{v_{i}^{\star 2}}\left(v_{i}^{\star 2}-\left\|v_{i}\right\|^{2}\right)\left\|v_{i}\right\| .
\end{aligned}
$$

On the contrary, $\kappa=0$ yields the resistive droop characteristics $(V-P, \omega-Q)$ which is similar to that of VOC [11]. It is noteworthy that the system is provably stable when $\kappa=$ $\tan ^{-1}\left(\frac{\omega_{0} L}{R}\right)$, where the inductance/resistance ratio is assumed constant across all transmission lines [19]. We notice both in simulation and in our experimental result that the system remains stable even when this assumption does not hold. By approximating $\left\|v_{i}\right\| \approx v_{i}^{\star}$, i.e., assuming a small voltage magnitude deviation, a more intuitive droop characteristic can be derived from (6) as

$$
\begin{aligned}
\frac{\mathrm{d}}{\mathrm{d} t} \theta_{i}=\omega_{i} & \approx \omega_{0}+\frac{\eta}{v_{i}^{\star 2}}\left(p_{i}^{\star}-p_{i}\right), \\
\frac{\mathrm{d}}{\mathrm{d} t}\left\|v_{i}\right\| & \approx \frac{\eta}{v^{\star}}\left(q_{i}^{\star}-q_{i}\right)+\eta \alpha\left(v_{i}^{\star}-\left\|v_{i}\right\|\right) .
\end{aligned}
$$

Note that at steady-state, (8) becomes

$$
\left\|v_{i}\right\| \approx v_{i}^{\star}+\frac{1}{\alpha v_{i}^{\star}}\left(q^{\star}-q\right) .
$$

To illustrates the droop relationships of dVOC, Fig. 2 displays an example design with $p^{\star}=0.5$ p.u., $q^{\star}=0$ p.u., $\eta=$ $43.43 \Omega \mathrm{rad} / \mathrm{sec}$ and $\alpha=0.9722 \mho$ and $\kappa=\pi / 2$. As expected from (6), dVOC droop features voltage dependent $\omega-P$ droop, which is different from conventional droop, and non-linear $V-Q$ droop with reduced voltage droop by $q$ change. We emphasize that dVOC has a local droop characteristic while guaranteeing that network of inverters controlled by dVOC (almost) globally converges to a pre-defined solution of the $\mathrm{AC}$ power flow equations.

\section{IMPLEMENTATION OF DVOC AND EXPERIMENTAL VALIDATION}

To verify the concept of dVOC, a hardware testbed shown in Fig. 3 was built. The specifications of the inverter hardware and dVOC parameters are tabulated in Table I. The reactive power set-point, $q^{\star}=-125$ var was set to incorporate the internal reactive power consumption by the filter capacitor $C_{f}$. As illustrated in Fig 3, the testbed was constructed to emulate 


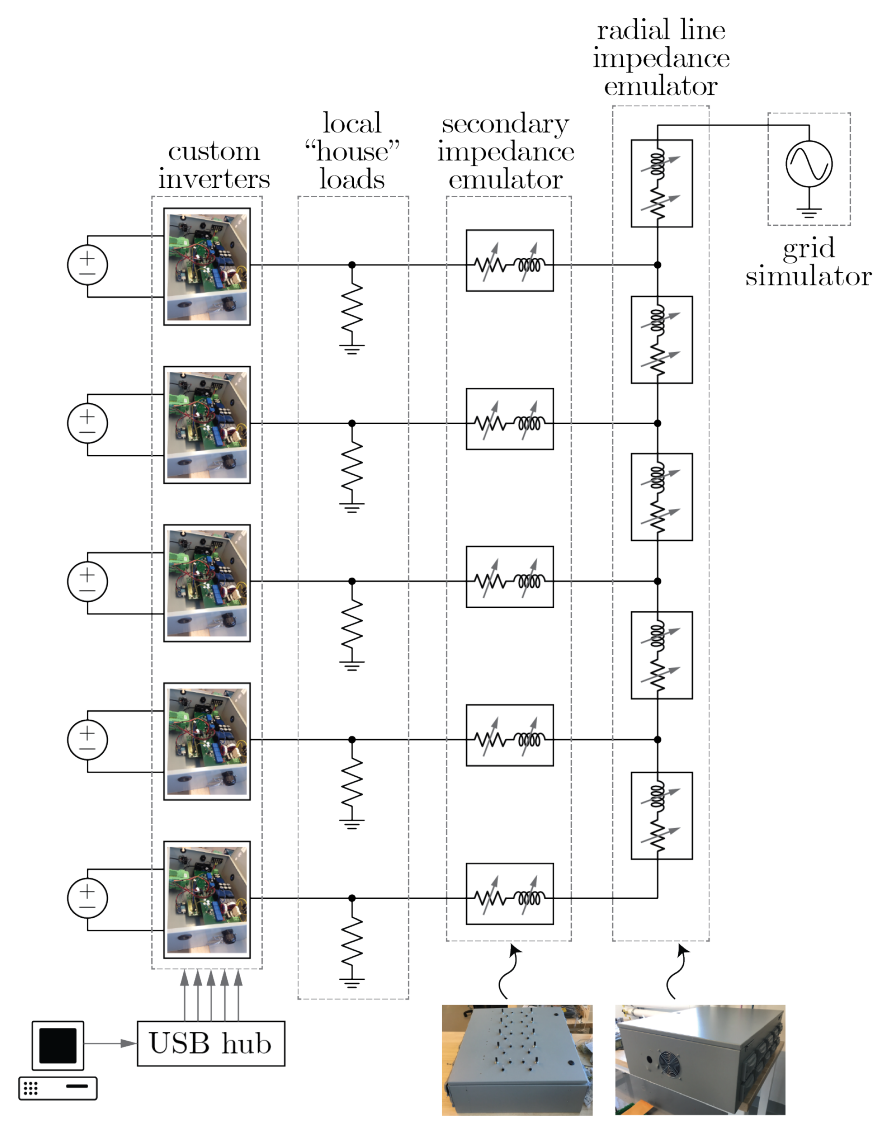

Fig. 3. Inverter-dominant grid testbed developed at NREL: Up to five inverters can be connected through a fully configurable impedance emulator to either a load or a grid simulator. For all experiments presented in this paper we consider two inverters connected in parallel to supply a resistive load. The full potential of the experimental setup will be exploited as part of current and future work.

TABLE I

DESIGN PARAMETERS FOR THE EXPERIMENTAL VALIDATION.

\begin{tabular}{cc}
\hline & dVOC Inverter parameters \\
\hline Item & Design Selection \\
\hline oscillator param. & $\eta=21.71 \Omega \mathrm{rad} / \mathrm{sec}, \alpha=0.9722 \mho, \kappa=\pi / 2$ \\
set-points & $p^{\star}=500 \mathrm{~W}, q^{\star}=-125$ var, $v^{\star}=120 V_{\mathrm{rms}}$ \\
controller & $320 \mathrm{~F} 28379 \mathrm{D}$, Texas Instruments \\
switching freq. & $32 \mathrm{KHz}$ \\
rated power & $1 \mathrm{VA}$ \\
filter params. & $L_{f}=1 \mathrm{mH}, C_{f}=24 \mu \mathrm{F}, L_{g}=0.2 \mathrm{mH}$ \\
\hline \hline
\end{tabular}

different system condition such as grid connected and islanded conditions with different distribution/transmission line impedances with up to five 1-kVA inverters. A test scenario was designed to verify collective grid regulation with multiple dVOC inverters. This section provides experimental results for

i) black start operation using a dVOC inverter from dead grid under loaded condition,

ii) dynamic synchronization and load sharing of the inverters under loaded condition,

iii) load transient performance with two inverters active,
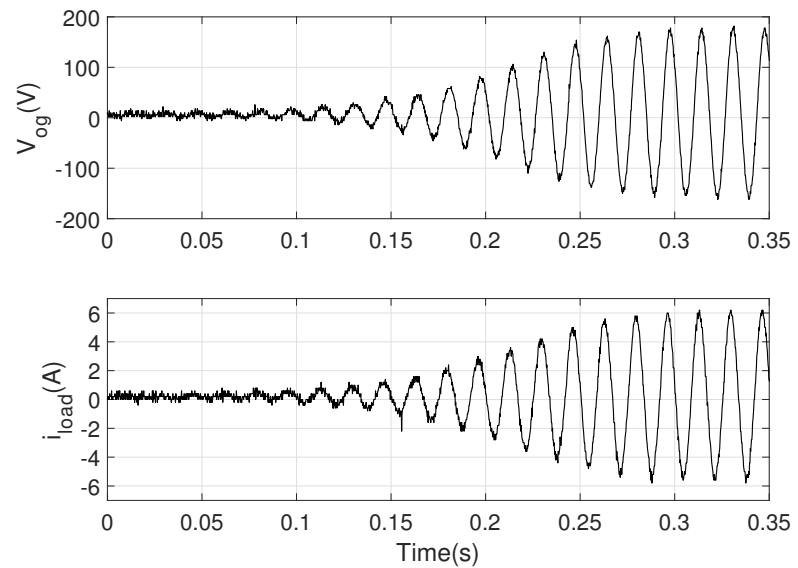

Fig. 4. Black start of inverter \#1 under $500 \mathrm{~W}$ load. dVOC with $p^{\star}=$ $500 \mathrm{~W}, q^{\star}=-125$ var is used to black-start the grid. Since 0 is an unstable equilibrium for $\mathrm{dVOC}$, measurement noise is enough to drive the voltage away from 0 to the nontrivial sinusoidal solution.

iv) real-time set-point update (dispatch) operation.

\section{A. Black Start}

Black start capability of grid forming inverters is a critical component for restoration after blackout to secure system resiliency in inverter-dominated power systems. Using a virtual oscillator, dVOC inverters can black-start a grid. Fig. 4 shows that inverter \#1 initiates-i.e., black starts-the grid under 500W resistive load; the grid voltage is gradually established by the oscillator as expected. Due to the reactive components, i.e., the LCL filter connected to the load side in the system representing the backbone of an electric power system, the inverter \#1 black starts the grid under $500 \mathrm{~W}$ and 250 var (reactive components from two LCL filters) load condition. $\mathrm{dVOC}$ is designed such that the synchronization dynamics are much faster than the voltage dynamics. This implies that, if we consider (6) during black-start the second term dominates i.e.,

$$
\frac{\mathrm{d}}{\mathrm{d} t}\left\|v_{i}\right\| \approx \frac{\eta \alpha}{v_{i}^{\star 2}}\left(v_{i}^{\star 2}-\left\|v_{i}\right\|^{2}\right)\left\|v_{i}\right\| .
$$

whose solution is given by

$$
\left\|v_{i}(t)\right\| \approx \frac{v_{i}^{\star} h_{0} \mathrm{e}^{\eta \alpha t}}{\sqrt{h_{0}^{2} \mathrm{e}^{2 \eta \alpha t}+1}},
$$

where

$$
h_{0}:=\frac{\left\|v_{i}(0)\right\|}{\sqrt{\left|\|v(0)\|^{2}-v_{i}^{2 \star}\right|}} .
$$

The derivation of (10) is provided in the Appendix. Equation (10) gives an indication of the black-start evolution of the voltage and can be used to predict the rise-time of the voltage magnitude. Fig. 5 shows a comparison of (10) with the experimental setup during the black start of inverter \#1. 


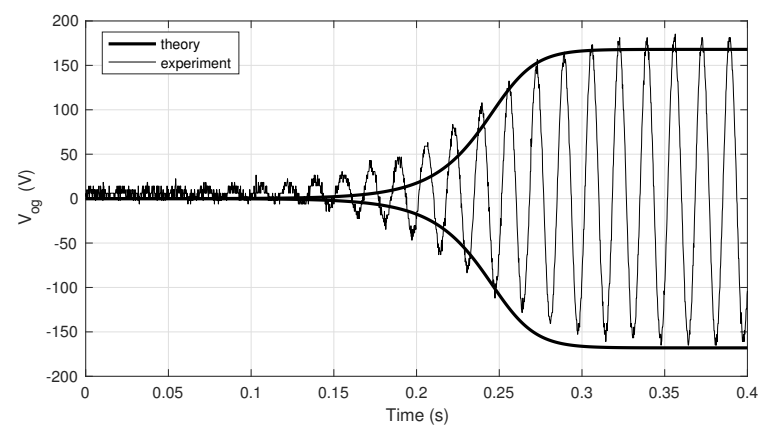

Fig. 5. Comparison of the theoretical and experimental curve for the voltage magnitude during black start of inverter \#1 under $500 \mathrm{~W}$ load.

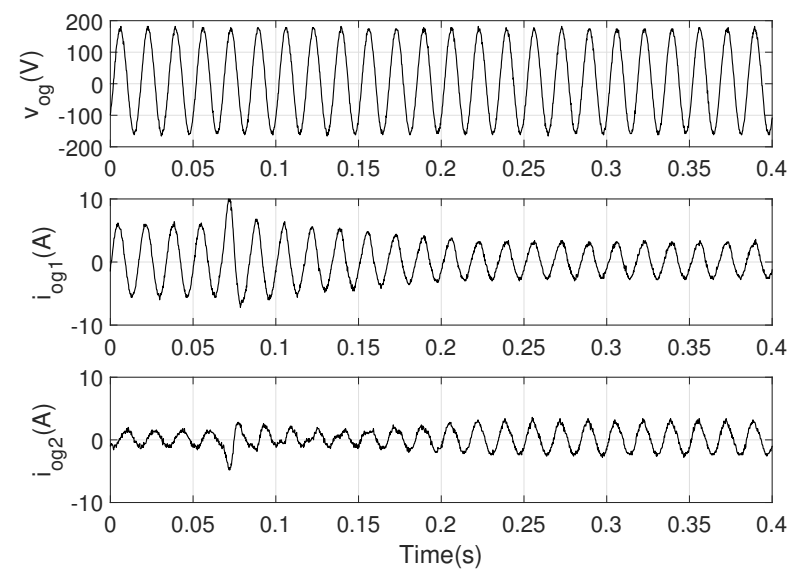

Fig. 6. Connecting inverter \#2 at $t=0 \mathrm{sec}$ while inverter \#1 is regulating the grid under $500 \mathrm{~W}$ load. $p^{\star}=500 \mathrm{~W}$ for inverter $\# 1$ and $\# 2$.

\section{B. Synchronization of Multiple Inverters and Load Transients}

As the electric grid is encountering frequent addition and subtraction of variable inverter-based renewable energy resources without significant system inertia from synchronous machines, the inverter controller should be capable of dynamic synchronization e.g., dynamic voltage and frequency regulation and load sharing. To verify these critical performance of grid-forming inverters, the experiment in Fig. 6 demonstrates a dynamic response of two grid-forming inverters; an inverter (\#2) is added to the grid at $t=0 \mathrm{sec}$ while the other $(\# 1)$ is maintaining the grid. It verifies i) synchronization of multiple inverters and ii) dynamic load sharing. As the inverter \#2, whose LCL filter is fed by inverter \#1 before the transient (i.e., the LCL filter acts as a capacitive load), is added to the system, the two inverters synchronize within $150 \mathrm{~ms}$ (10 cycles) without significant over-current and even load sharing (same $p^{\star}$ set-points). Fig. 7 displays operation under a load transient with two inverters active to collectively regulate the grid. Significantly different from the slow dynamic performance of conventional droop-controlled inverter operation, dVOC enables almost instantaneous dynamic load sharing without current overshoot and long settling time.
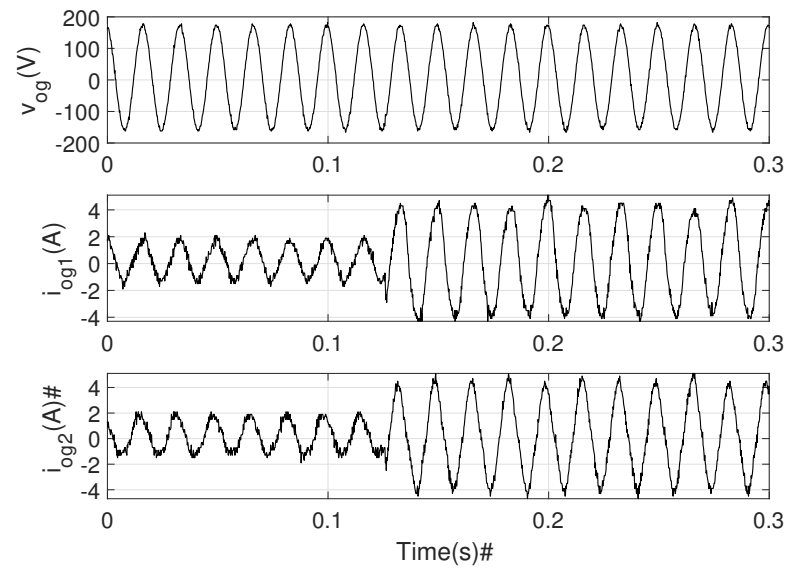

Fig. 7. $250 \mathrm{~W}$ to $750 \mathrm{~W}$ transient with two inverters active. $p^{\star}=500 \mathrm{~W}$ for inverter \#1 and \#2.

\section{Set-point Updates}

Dynamic set-point dispatch has received much attention due to the need for real-time power flow optimization. In an inverter-dominant grid with pervasive renewable generation, the benefit of real-time dispatch-ability will be even more striking than it is today. Because of the increased variability in generation capacity of renewable sources there will be a need of using the available resources and storage devices optimally. In Fig. 8 we demonstrate a power set-point update during operation of dVOC controllers. The aim was to reconfigure the power generation profile, e.g. to optimize the power flow. As inverter \#2 changes its active power set-point $p^{\star}$ from $250 \mathrm{~W}$ to $500 \mathrm{~W}$, the load sharing between Inverters \#1 and \# 2 is changed from $375 \mathrm{~W}: 375 \mathrm{~W}$ to $250 \mathrm{~W}: 500 \mathrm{~W}$. In addition, the grid frequency is recovered to the nominal $60 \mathrm{~Hz}$ since the loading condition matches to the nominal generation, verifying that the system can be reconfigured in real time. The ability of reconfiguring the power flow makes dVOC an extremely promising solution for an inverter-dominant system.

\section{CONCLUSION AND OUTLOOK}

This paper discussed the dVOC strategy and verified its grid-forming functionality in an inverter-dominant electric power grid. Using only local information, the dVOC inverters achieve almost instantaneous dynamic synchronization and load sharing. The analysis also verified the dVOC's embedded nonlinear droop law and how the inverters can be dispatched to optimize the power flow with programmable set-points. Synchronization and dispatchability were then verified by experimental results on a custom-built hardware setup, hinting that $\mathrm{dVOC}$ is a promising candidate for future grid applications.

\section{APPENDIX}

In the following we show that (10) solves the differential equation

$$
\frac{\mathrm{d}}{\mathrm{d} t}\left\|v_{i}\right\|=\frac{\eta \alpha}{v_{i}^{\star 2}}\left(v_{i}^{\star 2}-\left\|v_{i}\right\|^{2}\right)\left\|v_{i}\right\| .
$$



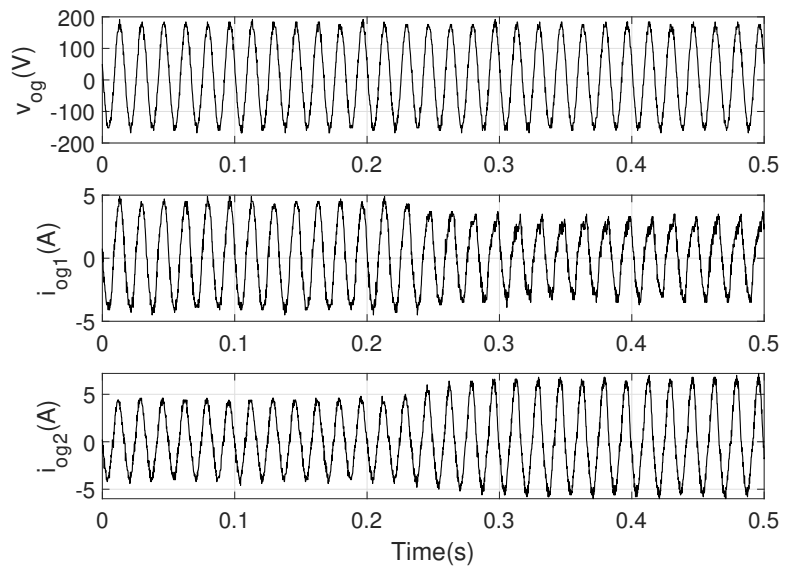

Fig. 8. Change of set-point: $p_{1}^{\star}$ of inverter \#2 updated from $250 \mathrm{~W}$ to $500 \mathrm{~W}$ and $p_{2}^{\star}=250 \mathrm{~W}$ unchanged for inverter \#1.

Making the change of variable $y=\frac{\left\|v_{i}\right\|}{v_{i}^{\star}}$ we obtain

$$
\frac{\mathrm{d}}{\mathrm{d} t} y=\eta \alpha\left(y-y^{3}\right) .
$$

By solving

$$
\int_{y(0)}^{y(t)} \frac{1}{y-y^{3}} \mathrm{~d} y=\eta \alpha \int_{0}^{t} \mathrm{~d} \tau
$$

and taking the exponent of both sides we obtain

$$
\frac{|y(t)|}{\sqrt{\left|y^{2}(t)-1\right|}}=\frac{|y(0)|}{\sqrt{\left|y^{2}(0)-1\right|}} \mathrm{e}^{\eta \alpha t},
$$

by inverting the left-hand side in the interval $[0,1]$ and changing the variable back to $\left\|v_{i}\right\|$ we obtain (10).

\section{ACKNOWLEDGEMENTS}

The authors would like to thank Dr. Miguel Rodriguez and Mr. Ramanathan Thiagarajan for their assistance in the inverter hardware development.

\section{REFERENCES}

[1] "World Energy Outlook-Part B: Special focus on Renewable Energy," tech. rep., International Energy Agency, 2016.

[2] P. Beiter, M. Elchinger, and T. Tian, "Renewable energy data book," US Department of Energy's National Renewable Energy Laboratory (NREL), 2016.

[3] L. A. Tôrres, J. P. Hespanha, and J. Moehlis, "Synchronization of identical oscillators coupled through a symmetric network with dynamics: A constructive approach with applications to parallel operation of inverters," IEEE Transactions on Automatic Control, vol. 60, no. 12, pp. 3226-3241, 2015.

[6] B. Kroposki, B. Johnson, Y. Zhang, V. Gevorgian, P. Denholm, B.-M. Hodge, and B. Hannegan, "Achieving a 100\% renewable grid: Operating electric power systems with extremely high levels of variable renewable energy," IEEE Power and Energy Magazine, vol. 15, no. 2, pp. 61-73, 2017.
[4] C. Arghir, T. Jouini, and F. Dörfler, "Grid-forming control for power converters based on matching of synchronous machines," Automatica, vol. 95 , pp. 273-282, 2018.

[5] J. Liu, Y. Miura, T. Ise, et al., "Comparison of dynamic characteristics between virtual synchronous generator and droop control in inverterbased distributed generators," IEEE Trans. Power Electron, vol. 31, no. 5, pp. 3600-3611, 2016.

[7] M. C. Chandorkar, D. M. Divan, and R. Adapa, "Control of parallel connected inverters in standalone AC supply systems," IEEE Transactions on Industry Applications, vol. 29, no. 1, pp. 136-143, 1993.

[8] V. Mariani, F. Vasca, J. C. Vásquez, and J. M. Guerrero, "Model order reductions for stability analysis of islanded microgrids with droop control," IEEE Transactions on Industrial Electronics, vol. 62, no. 7, pp. 4344-4354, 2015.

[9] J. W. Simpson-Porco, F. Dörfler, and F. Bullo, "Voltage stabilization in microgrids via quadratic droop control," IEEE Transactions on Automatic Control, vol. 62, no. 3, pp. 1239-1253, 2017.

[10] M. C. Chandorkar, D. M. Divan, and R. Adapa, "Control of parallel connected inverters in standalone ac supply systems," IEEE Transactions on Industry Applications, vol. 29, no. 1, pp. 136-143, 1993.

[11] B. Johnson, M. Rodriguez, M. Sinha, and S. Dhople, "Comparison of virtual oscillator and droop control," in IEEE Workshop on Control and Modeling for Power Electronics, 2017.

[12] Q.-C. Zhong and G. Weiss, "Synchronverters: Inverters that mimic synchronous generators," IEEE Transactions on Industrial Electronics, vol. 58, no. 4, pp. 1259-1267, 2011.

[13] T. Jouini, C. Arghir, and F. Dörfler, "Grid-friendly matching of synchronous machines by tapping into the DC storage," in IFAC Workshop on Distributed Estimation and Control in Networked Systems, pp. 192197, 2016.

[14] S. D'Arco and J. A. Suul, "Virtual synchronous machinesClassification of implementations and analysis of equivalence to droop controllers for microgrids," IEEE PowerTech, 2013.

[15] RG-CE System Protection \& Dynamics Sub Group, "Frequency stability evaluation criteria for the synchronous zone of continental Europe," tech. rep., ENTSO-E, 2016.

[16] M. Sinha, F. Dörfler, B. Johnson, and S. Dhople, "Uncovering droop control laws embedded within the nonlinear dynamics of van der pol oscillators," IEEE Transactions on Control of Network Systems, vol. 4, no. 2, pp. 347-358, 2017.

[17] B. B. Johnson, S. V. Dhople, A. O. Hamadeh, and P. T. Krein, "Synchronization of parallel single-phase inverters with virtual oscillator control," IEEE Transactions on Power Electronics, vol. 29, no. 11, pp. 6124-6138, 2014.

[18] M. Colombino, D. Groß, and F. Dörfler, "Global phase and voltage synchronization for power inverters: a decentralized consensus-inspired approach," in IEEE Conference on Decision and Control, 2017.

[19] D. Groß, M. Colombino, J.-S. Brouillon, and F. Dörfler, "The effect of transmission-line dynamics on grid-forming dispatchable virtual oscillator control," arXiv preprint arXiv:1802.08881, 2018.

[20] M. Colombino, D. Groß, J.-S. Brouillon, and F. Dörfler, "Global phase and magnitude synchronization of coupled oscillators with application to the control of grid-forming power inverters," Submitted to IEEE Transactions of Automatic Control, avialable online: arXiv:1710.00694, 2018.

[21] B. B. Johnson, M. Sinha, N. G. Ainsworth, F. Dörfler, and S. V. Dhople, "Synthesizing virtual oscillators to control islanded inverters," IEEE Transactions on Power Electronics, vol. 31, no. 8, pp. 6002-6015, 2016. 